\title{
Fever, Pancreatitis, Gastrointestinal Bleeding, Multi Organ Dysfunction Syndrome Due To Enteric Fever - A Case Report With Review of Literature
}

\author{
Authors \\ Dr C R Khatua*, Dr Sooraj Menon R*, Dr Roniya Simethy ${ }^{\#}$, Dr Kumar Avijeet Dash ${ }^{\#}$ \\ Dr Vishnu $\mathrm{K}^{\#}$ \\ *Assistant Professor, \#Post Graduate Student \\ Department of Medicine, MKCG Medical College
}

\begin{abstract}
The diagnosis of enteric fever requires a high degree of clinical suspicion as the signs and symptoms of this disease can be confused with other diseases. Typhoid is still a major health problem in Asian countries, especially in India. Here we present a case of a 23-year old male who had presented with fever along with other rare manifestations like GI bleeding, pancreatitis, myositis and multi organ involvement. As patient may present with rare clinical features, high clinical suspicion is the corner stone of diagnosis.
\end{abstract}

Keywords: Enteric fever, Salmonella typhi and paratyphi, GI bleeding and intestinal perforation, Pancreatitis.

\section{INTRODUCTION}

Enteric fever is a systemic disease characterised by fever, abdominal pain, headache and other constitutional symptoms, caused by dissemination of $S$. typhi or $S$. paratyphi ${ }^{1,2}$. It can be caused by salmonella infection like S. typhi or S. paratyphi serotypes A, B, and C. When caused by S. typhi, it is known as typhoid fever. Typhoid fever has high incidence in five Asian countries, i.e. India, Pakistan, Indonesia, Vietnam and China ${ }^{4}$.Infection is transmitted by consumption of fecal contaminated food or drink by ill or asymptomatic chronic carriers. Sexual transmission between male partners and infection of health care workers from infected patients, specimens and cultures have been reported ${ }^{1}$. High incidence of enteric fever correlates with poor sanitation and lack of access to clean drinking water and in endemic regions, it is more prevalent in urban areas ${ }^{1}$.

\section{CASE REPORT}

A young male of 23years was admitted to our hospital with fever, headache, myalgia, pain abdomen, vomiting and yellow discoloration of urine and conjunctiva for 15days. On admission he was confused to verbal commands, febrile $\left(102^{\circ} \mathrm{F}\right)$ and other clinical examination revealed pallor, icterus, subconjuctival hemorrhage, bilateral parotid swelling with pulse rate-96/min, BP-100/60mmHg. Patient was tachypnoeic with a respiratory rate of $24 /$ minute. Per abdominal examination showed tender hepatomegaly $(3 \mathrm{~cm}$ 
below right costal margin), right intercostal tenderness and splenomegaly $(2 \mathrm{~cm})$. On $2^{\text {nd }}$ day he developed distension of abdomen and melena. Examination of respiratory and cardiovascular system revealed normal findings. His labs showed Hemoglobin of $10.2 \mathrm{~g} / \mathrm{dl}$, WBC count of $13.2 \times 10$ $3 / \mathrm{cmm}$ with Neutrophil-84\%,Lymphocytes-13\%, total platelet count(TPC) $2.41 \mathrm{akh} / \mathrm{cmm}$, ESR was $20 \mathrm{~mm}$ in first hour, serum creatinine was 1.7 $\mathrm{mg} / \mathrm{dL}$, blood urea of $144 \mathrm{mg} / \mathrm{dl}$ but increased to $5.8 \mathrm{mg} / \mathrm{dl}$ and $187 \mathrm{mg} / \mathrm{dl}$ respectively on $3^{\text {rd }}$ day, $\mathrm{Sr} \mathrm{Na}^{+}-125$, Sr K $\mathrm{K}^{+}-2.5$, liver function test showed total bilirubin-3.3, direct bilirubin-1.7,AST-537, ALT-251, ALP-536 IU/L and MP (slide, ICT both antigen and antibody), HBs Ag, anti HCV,HIV tests were negative. Serum amylase (403) and lipase (548) were raised, CPK was 1077, stool occult blood was positive, widal test was positive in 1:320 titer for S.typhi and S.paratyphi and also Typhidot test(Ig G on $15^{\text {th }}$ day of admission) was positive. On $4^{\text {th }}$ day hemoglobine decreased to 6.8 from $10.2 \mathrm{gm} / \mathrm{dl}$. One of our differential diagnoses, Leptospirosis, was excluded by negative antibody test and normal urine $\mathrm{Mg}^{++}$test, $\mathrm{X}$-ray chest was normal. Ultrasound of abdomen showed hepatomegaly and splenomegaly. We started treatment for enteric fever with Injection ceftriaxone (2gm/day), Tab azithromycine (1gm/day), Injection pantoprazole, Injection tranexamic acid, blood transfusion(5 units), haemodialysis 3 settings, Injection potassium infusion for hypokalemia and Injection torsemide. Gradually patient improved clinically with treatment he became afebrile, melena stopped, urination increased to 2.8 to $3 \mathrm{~L} /$ day without any diuretics and serum urea and creatinine became normal and was discharged on $24^{\text {th }}$ day of admission. On follow up after two weeks, patient was asymptomatic with normal hemogram, liver function test and renal function test.

\section{DISCUSSION}

Enteric fever is caused by infection with Salmonella and begins with consumption of fecal contaminated food and water. Incubation period averages about 10 -14 days. Salmonella is an intracellular pathogen; infection causes breach in the mucosal epithelium of intestine by transcytosis. Having crossed the epithelial barrier, organisms invade and replicate in macrophages in Peyer patches, mesenteric lymph nodes, and spleen. Serotypes other than typhi usually do not cause invasive disease as they lack human-specific virulence facor. Following replication, bacteraemia occurs and disseminates to other organs like lungs, gallbladder, kidneys, or central nervous sysem ${ }^{2}$. During prodermal stage, there is increasing headache, myalgia, arthralgia, abdominal pain, vomiting and diarrhoea more commonly than constipation ${ }^{1,2}$. Fever is documented in $>75 \%$ of cases, abdominal pain in $30-40 \%$ cases, so high index of suspicion is needed for diagnosis in patients with fever and recent travel history. Prolonged fever (4wk) is most prominent symptom, it ascends in a stepwise fashion till 7 to 10 days, and then it reaches a plateau $^{2}$. Physical findings include rose spots, hepatosplenomegaly, epistaxis, relative bradycardia, coated tongue.

Complications occur in about $30 \%$ of untreated cases and account for $75 \%$ of deaths.GI bleeding (10-20\%) and intestinal perforation most commonly occur in 3rd or 4th week of illness and results from hyperplasia, ulceration, necrosis of peyers patches at the initial site of salmonella infiltration. Neurological manifestations (2-40\%) include meningitis, Guillain-Barre Syndrome, neuritis, neuropsychiatric symptoms like muttering delirium. Rare complications of enteric fever include disseminated intravascular coagulation (DIC), pancreatitis, parotitis, glomerulonephritis, haemolytic-uremic syndrome (HUS), pericarditis, myocarditis, endocarditis, endophthalmitis $^{1,2}$. It has been stated that hemorrhagic pancreatitis rarely occurs in association with typhoidal infections. More recent reviews of typhoid fever and its complications have much not mentioned about pancreatitis but earlier several reports of confirmed or probable pancreatitis with typhoid fever were published ${ }^{3}$. 
But in our case the prominent features were fever, jaundice, GI bleeding, pancreatitis, myositis, acute renal failure and CNS manifestations, which indicate atypical presentations are not rare now days.

Diagnosis requires suspicion of febrile traveler returning from a developing region of the world. Other than a positive blood culture no specific lab test is diagnostic of enteric fever. Leucopenia and neutropenia are detectable in $15-25 \%$ cases but leucocyosis with neutrophilia is present when complicated with intestinal perforation and secondary infection (Our patient had leucocytosis with neurtophilia) ${ }^{1,4}$. Moderately elevated level of LFT and muscle enzyme levels are present in some cases. BM culture and stool culture gave higher yield than blood culture alone. Serological test include classic widal test for febrile agglutinins and rapid test for antibody (IgM \& $\mathrm{IgG})$ against outer membrane protein or (O: 9) antigen ${ }^{1}$. Recently newer investigations like IDL Tubex which detect IgM and new typhoid IgM dipstick assay are available with high sensitivity and positive predictive value ${ }^{4}$.

\section{CONCLUSION}

Typhoid is a systemic disease caused by Salmonella typhi. The diagnosis is made on clinical grounds for which high clinical suspicion, assisted with rapid quick laboratory tests are required to differentiate from diseases which mimic similar symptoms like malaria, leptospirosis, sepsis with other bacterial pathogens and viral infections like dengue and acute hepatitis $^{4,5}$. However, safe water supply, proper sanitation, early diagnosis and treatment, and effective vaccination are important measures to control typhoid.

\section{REFERENCES}

1. David A Pegues, Samuel I. Miller. Salmonellosis: Harrison's Principles of Internal Medicine.19 ${ }^{\text {th }}$ Edition.2015 ; 190):1049-1055.

2. Brian S. Schwartz. Bacterial \& Chlamydial Infections: Current Medical Diagnosis \& Treatment. 2016 ;(33):1443-1444.

3. Irwin J. Russell, Pierre Forgacs, Joseph E. Geraci, Pancreatitis Complicating Typhoid Fever Report of a Case. JAMA. 1976;235(7):753-754.

4. Uttam K Paul, Smarajit Banik. Typhoid Fever: Presentation and Management Strategies in 2016: Medicine Update 20161(Vol-2);(141):706-711.

5. Surendra Daga. Typhoid Fever: Overview with Recent Advances: Medicine Update 2016-1(Vol-2);(142):712-714. 